EPJ Web of Conferences 71, 00069 (2014)

DOI: 10.1051/epjconf/20147100069

(C) Owned by the authors, published by EDP Sciences, 2014

\title{
Femtoscopy at the LHC
}

\author{
Adam Kisiel ${ }^{1}$ \\ ${ }^{1}$ Faculty of Physics, Warsaw University of Technology, Koszykowa 75, 00-662 Warsaw, Poland
}

\begin{abstract}
We discuss the results of femtoscopic correlations performed by various experiments at the Large Hadron Collider and theoretical efforts to interpret them. Twoparticle low relative momentum correlations are used to measure the size of the region emitting particles in nuclear collisions. In $p p$ collisions it is important to test the spatiotemporal characteristics of particle production at small scales. The results are compared to sizes measured in $\mathrm{Pb}+\mathrm{Pb}$ collisions, as a function of pair momentum and centrality. They are interpreted in the frame of hydrodynamic models and used to test the hypothesis of the collective behavior of matter in such collisions.
\end{abstract}

\section{Introduction}

The Large Hadron Collider (LHC) is the largest particle accelerator in the world, located under the Franco-Swiss border near Geneva in the CERN laboratory. It has started operating in 2009 and has delivered $p p$ collisions at center-of-mass energies of $\sqrt{s}=0.9 \mathrm{TeV}, \sqrt{s}=2.76 \mathrm{TeV}, \sqrt{s}=7 \mathrm{TeV}$ and $\sqrt{s}=8 \mathrm{TeV}$ as well as $\mathrm{Pb}-\mathrm{Pb}$ collisions at a center-of-mass energy of $\sqrt{s_{\mathrm{NN}}}=2.76 \mathrm{TeV}$ and $\mathrm{p}-\mathrm{Pb}$ collisions at $\sqrt{s_{\mathrm{NN}}}=5.02 \mathrm{TeV}$. Two general purpose detectors operate at the LHC, CMS and ATLAS. The former has published results of femtoscopic correlations in $p p$ collisions [1]. ALICE [2], another experiment conducted at the LHC is dedicated to study heavy-ion collisions. Its main advantages are: tracking at low momentum and excellent particle identification (PID) capabilities at low and intermediate momentum. These characteristics make ALICE an ideal setup for femtoscopic measurements [3-6].

The femtoscopy technique is based on two-particle low relative momentum correlations. It has been used extensively in elementary and heavy-ion collisions [7-9]. In the former the produced system has the size of the order of a size of the proton. Most of the measurements have been done only in minimum-bias collisions, or as a function of final state particle multiplicity. Only STAR has measured the system size as a function of pair momentum [10]. No doubly-differential measurement has been done in elementary collisions.

In heavy-ion collisions the body of pre-LHC data is more rich. In particular pion femtoscopy as a function of pair momentum and centrality has been studied [11-13]. It allows a test of the linear scaling of the three-dimensional radii with $\left\langle d N_{c h} / d \eta\right\rangle^{1 / 3}$ as well as a measurement of the decrease of the size with pair momentum. Both trends are then interpreted in the frame of models assuming a collective behavior of matter, such as hydrodynamic with particular assumptions tailored to the properties of strongly interacting matter.If models describe all aspects of this data, it will give strong argument for the validity of the picture. 
In this work first the formalism of the femtoscopic measurement is introduced in Sec. 2. Then theoretical expectations for LHC femtoscopic measurements is presented in Sec. 3. The results for heavy-ion collisions and their interpretation is discussed in Sec. 4 . In Sec. 5 the results from $p p$ collisions are shown. The differences and similarities between the two dataset are pointed out and an outlook for future measurements is given.

\section{Femtoscopy measurement}

In the femtoscopic correlation measurement a two-particle correlation function is measured versus the pair invariant relative momentum $\vec{q}=\left|\overrightarrow{p_{1}}-\overrightarrow{p_{2}}\right|$. In the experiment it is defined as:

$$
C(\vec{q})=\frac{P\left(\overrightarrow{p_{1}}, \overrightarrow{p_{2}}\right)}{P\left(\overrightarrow{p_{1}}\right) P\left(\overrightarrow{p_{2}}\right)}
$$

that is a ratio of a conditional probability to observe a given pair of particles in the same event to the product of observing those particles independently. The uncorrelated background is constructed with the "mixing" technique, where the two particles are taken from two different events with similar multiplicity and other detector characteristics. For the relative momentum $\vec{q}$ one can consider only its magnitude $q_{i n v}$, which results in one dimensional correlation function. Then the radius $R_{i n v}$ is extracted from the fit with:

$$
C\left(q_{i n v}\right)=(1-\lambda)+K\left(q_{i n v}\right)\left[1+\lambda \exp \left(-R_{i n v}^{2} q_{i n v}^{2}\right)\right]
$$

where $\lambda$ is the fraction of correlated pairs in the sample. It also account for other factors, such as nongaussian form of the source and is usually close to 0.5 in experiment. $K\left(q_{i n v}\right)$ is the factor accounting for the Coulomb repulsion between pions, which results in a sharp decrease of correlation at very low $q$. Another possibility is to analyze the dependence in Longitudinally Co-Moving System (LCMS), where the longitudinal momentum of the pair vanishes. $\vec{q}$ is then decomposed into three components: long along the beam direction, out along the pair transverse momentum $k_{T}=\left|p_{T, 1}+p_{T, 2}\right| / 2$, and side perpendicular to the other two. The fitting formula then contains three radii: $R_{\text {out }}, R_{\text {side }}$ and $R_{\text {long }}$ and is as follows:

$$
C(\vec{q})=(1-\lambda)+K\left(q_{\text {inv }}\right)\left[1+\lambda \exp \left(-R_{\text {out }}^{2} q_{\text {out }}^{2}-R_{\text {side }}^{2} q_{\text {side }}^{2}-R_{\text {long }}^{2} q_{\text {long }}^{2}\right)\right] .
$$

The three radii can then be compared with theoretical predictions. The three-dimensional analysis is more demanding in terms of statistics, so up to now it could be realistically done for pions, and rarely for kaons.

\section{Theoretical predictions}

Particle production at low momentum (the so-called "soft sector") in heavy-ion collisions is understood in the frame of hydrodynamic models. The produced system is treated as continuos, behaving collectively. The simulations predict certain phenomena, most notably collective radial flow pushing the matter outwards from the center of the system, as well as its azimuthal oscillation called elliptic flow. The flow is a result of large initial temperature and pressure gradients. When moving from RHIC to LHC energies, the initial temperature grows. Since freeze-out occurs at a fixed temperature, the LHC system needs more time to cool down and naturally lives longer. It also develops stronger flows, as these reflect the time integral of pressure gradients. Less obviously some calculations show that the freeze-out pattern changes in LHC collisions from outside-in to inside-out [14]. 
All these features are reflected in pion femtoscopy measurements. The size of the system is reflected in the absolute value of the femtoscopi radii, which are expected to grow from RHIC to LHC. The radial flow phenomenon causes the so-called "lengths of homogeneity" effect $[15,16]$, that is the decrease of apparent system size with pair momentum. This decrease is expected to be more prounounced at LHC energies. Lastly the change of the freeze-out pattern produces a non-trivial effect of decreasing the ratio of outwards to sidewards transverse radii of the system [14]. The radius is also expected to be smaller for pairs of heavier mesons, like kaons, which naturally have higher average pair momentum.
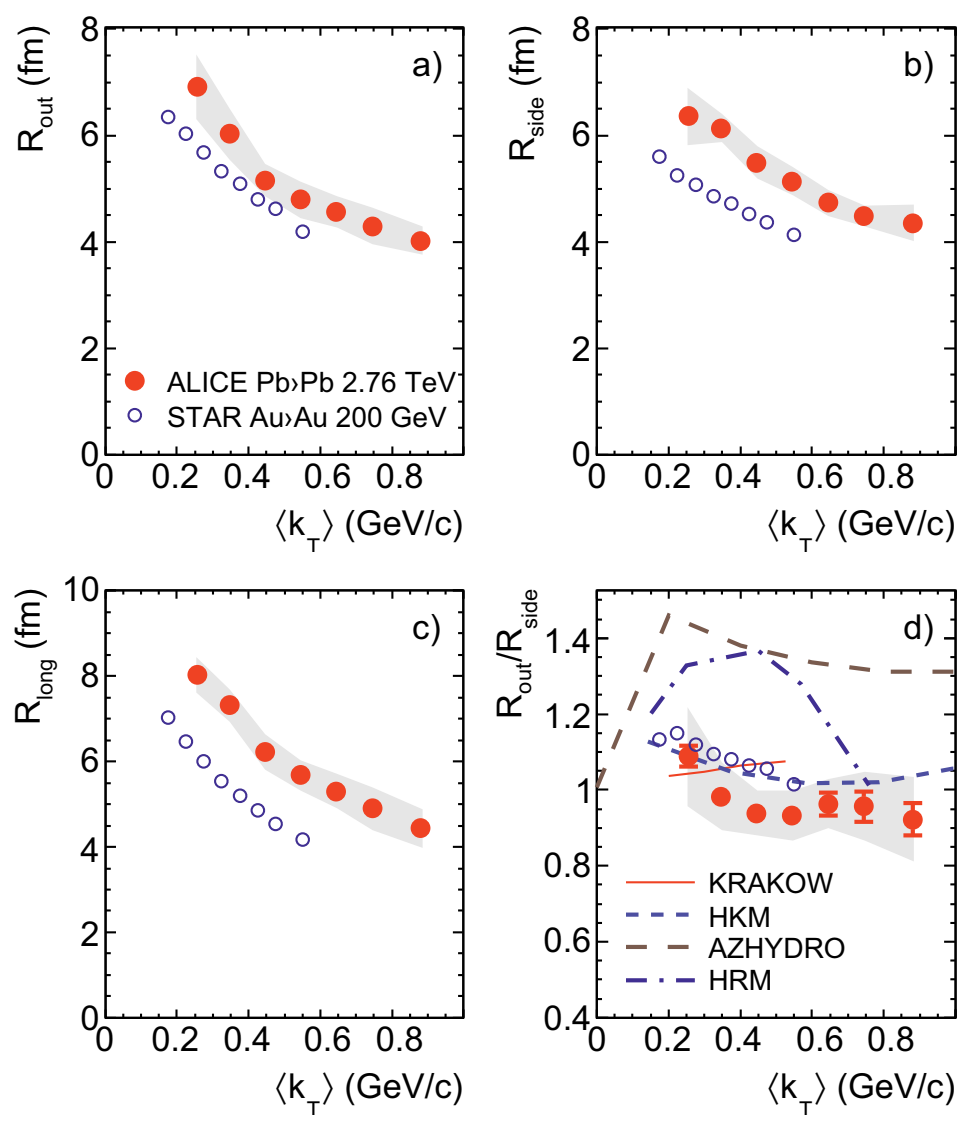

Figure 1. Pion femtoscopic measurement in central $\mathrm{Pb}-\mathrm{Pb}$ collisions at $\sqrt{s_{N N}}=2.76 \mathrm{ATeV}$ [5].

All the predictions above have been made after careful analysis of RHIC data. It showed that in hydrodynamic models certain features must be included in order to reproduce experimental radii [17]. In the initial stages of the collision, before matter thermalization (and the formation of Quark-Gluon Plasma) collective flow must be included (so-called "pre-thermal flow" assumption). The Equation of State (EOS) of the matter must not exhibit a first-order phase transition from Quark-Gluon Plasma to regular hadronic matter. Production of particles from strongly-decaying resonances and the hadronic rescattering must be considered. If models based on these assumptions continue to describe the data 
at the LHC, it is a strong argument for the correcness of the picture and also shows that LHC can serve as a laboratory to test the same QGP that has been observed at RHIC.

The predictions for femtoscopic measurements in $p p$ collisions at the LHC are limited. An interesting scaling of radii between $p p$ and $\mathrm{Au}+\mathrm{Au}$ collisions at various collision energies was reported by STAR [18]. These were however for minimum-bias $p p$ data only. A check for similar scaling at the LHC should be performed.

\section{Results for heavy-ion collisions}

The pion femtoscopic radii were measured in central $\mathrm{Pb}-\mathrm{Pb}$ collisions at $\sqrt{s_{N N}}=2.76 \mathrm{ATeV}$ by ALICE [5]. In particular the three-dimensional analysis in LCMS was performed. The results are shown in Fig. 1. As a comparison, results from top RHIC energy (from the STAR experiment) are also shown. All three radii are significantly larger at the LHC, which is qualitatively consistent with predictions. The slope of the $k_{T}$ dependence is similar or more steep as compared to RHIC, again compatible with expectations. The $R_{\text {out }} / R_{\text {side }}$ ratio shown in the fouth panel is smaller than at RHIC, showing that the non-trivial prediction from hydrodynamics of the change of freeze-out pattern is consistent with data.

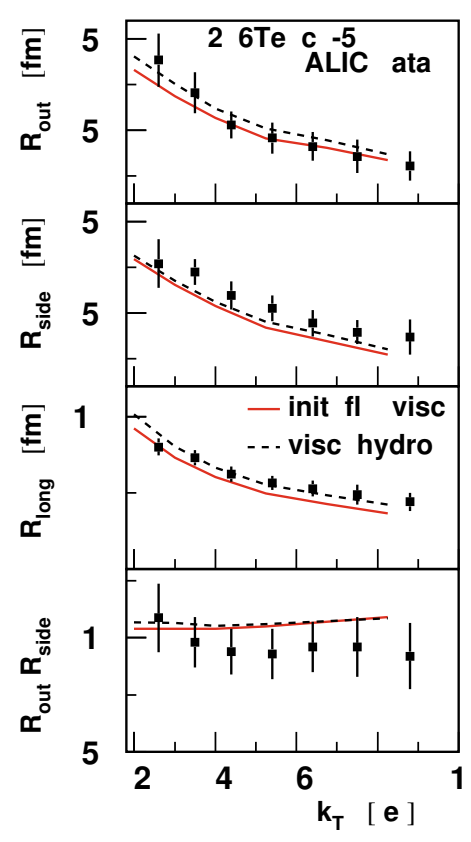

Figure 2. Hydrodynamic model description of pion femtoscopic measurement in central $\mathrm{Pb}-\mathrm{Pb}$ collisions at $\sqrt{s_{N N}}=2.76 \mathrm{ATeV}[19]$.

The data from LHC has motivated a dedicated search for correct model description of the system. In Fig. 2 the recent calculation from a modern hydrodynamic model [19] is presented. All major features of the data are reproduced, showing that the collective behaviour of matter in $\mathrm{Pb}-\mathrm{Pb}$ collisions at the LHC is well understood in this framework. 
The overall volume of the system created in a central $\mathrm{Pb}-\mathrm{Pb}$ collisions can be inferred from the data. It is a factor of 2 larger than the highest measured at RHIC. From the dependence of the longitudinal radius on pair momentum one can also deduce the total duration of the system evolution, from the collision until freeze-out. It is estimated to be on the order of $13 \mathrm{fm} / c, 30 \%$ larger than at RHIC. Therefore the QGP is bigger at the LHC and lives longer, so its imprint on other observables, such as jet quenching should be more pronounced.

The radii can also be compared to the scaling with $\left\langle d N_{c h} / d \eta\right\rangle^{1 / 3}$ observed for lower energies [9]. The longitudinal radius is in agreement with the scaling. $R_{\text {side }}$ is consistent within large systematic uncertainty, but $R_{\text {out }}$ is below the line determined by lower energy points. This violation of the simple scaling is the reflection of the hydrodynamic prediction of the change of the freeze-out pattern. It is a proof that naive scaling arguments are not general and that the initial state parameters such as collision energy do influence the observed femtoscopic radii.

\section{Results for $p p$ collisions}
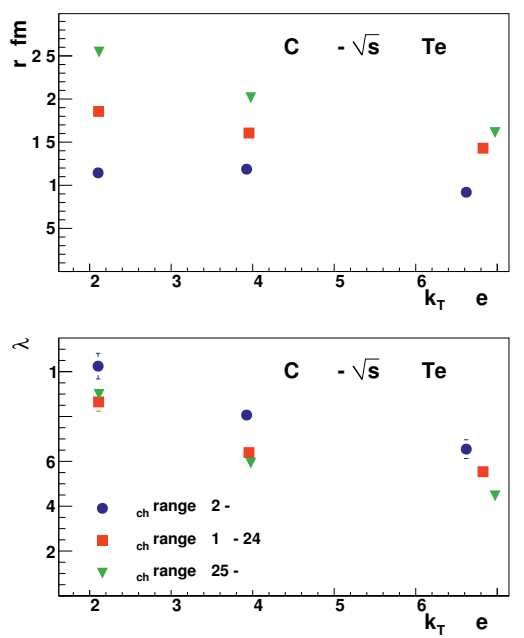

Figure 3. Pion femtoscopic radii as a function of multiplicity and pair momentum, measured by CMS [1].

Two-pion femtoscopic correlations have been measured in $p p$ collisions and other elementary reaction at various collision energies [7, 8]. They give insight into the space-time characteristics of particle production in such systems. They are also an important baseline for the interpretation of the heavy-ion results. There are also claims of universal scaling of the HBT radii with $\left\langle d N_{c h} / d \eta\right\rangle^{1 / 3}$, which could include all systems, regardless of the initial size [9]. The data from LHC has motivated a dedicated search for correct model description.

At the LHC the measurement for $p p$ collisions has been performed by CMS at $\sqrt{s_{N N}}=0.9$ and $7 \mathrm{TeV}$ [1]. The results for one dimensional radii are shown in Fig. 3. They show that the radius grows 
with the final state multiplicity of the collision. They also indicate that the radius dependence on pair transverse momentum $k_{\mathrm{T}}$ changes with multiplicity. At low multiplicity it is relatively independent of $k_{\mathrm{T}}$, while for higher multiplicity it is significantly decreasing with $k_{\mathrm{T}}$. The latter trend is reminiscent of the behavior of radii in heavy-ion collisions, therefore it is worth investigating in detail. It has also been determined that the shape of the correlation function is not well described by the Gaussian assumption and an exponential shape is more adequate.

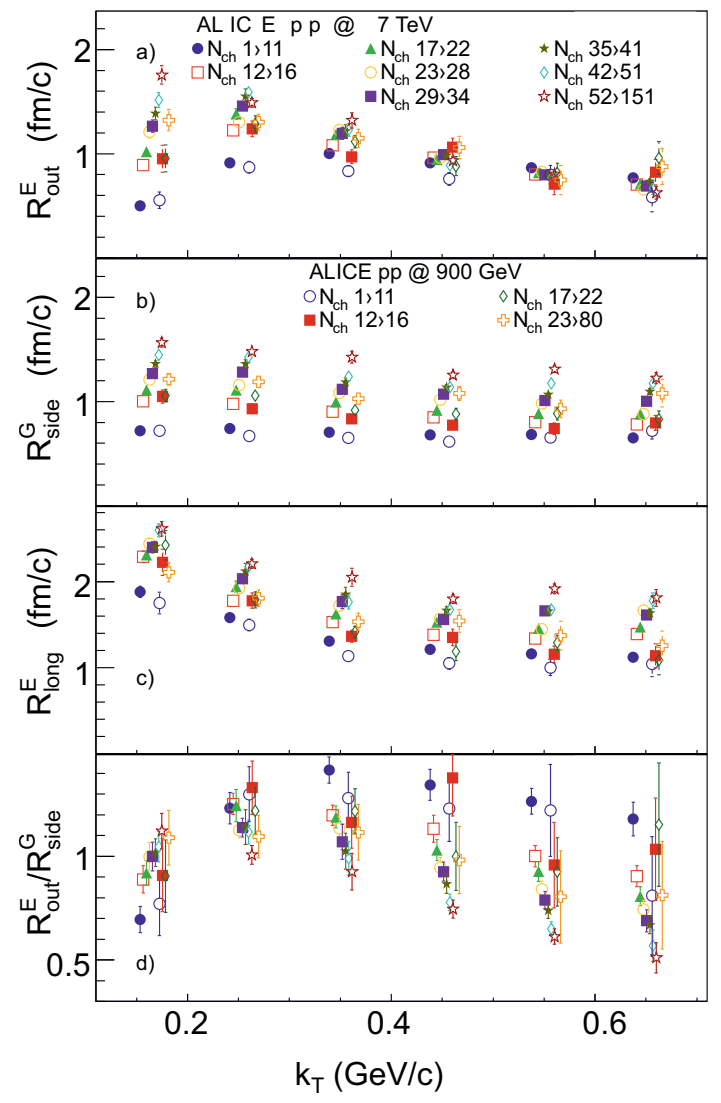

Figure 4. Pion femtoscopic radii as a function of $\sqrt{s}$, event multiplicity and pair momentum, measured by ALICE [4].

ALICE has performed a detailed investigation of femtoscopy in $p p$ collisions $[3,4]$. It has measured the correlations as a function of collision energy, $\left\langle d N_{c h} / d \eta\right\rangle^{1 / 3}$ and $k_{\mathrm{T}}$. It has also performed the analysis in three dimensions in LCMS, similarly to the analysis in heavy ion collisions discussed above. As a result a detailed investigation and direct comparisons to other measurements was possible. The resulting radii are shown in Fig. 4. The plotted values correspond to the three-dimensional source shape, which is Gaussian in side and exponential in out and long, confirming the finding of CMS, that the emitting shape is not Gaussian. However, for comparisons with data in other systems, most importantly with heavy-ion collisions a Gaussian fit was performed as well [4].

The results for two collision energies, $\sqrt{s}=0.9$ and $7 \mathrm{TeV}$ are in agreement with each other, if taken at the same $\left\langle d N_{c h} / d \eta\right\rangle^{1 / 3}$ and $k_{\mathrm{T}}$. This finding is confirmed with direct investigation of the raw 
correlation function which is comparable within statistical errors at both energies. This would support an argument that $\left\langle d N_{c h} / d \eta\right\rangle^{1 / 3}$ is the relevant variable for femtoscopic radii scaling, at least for the same colliding system at various energies.

The radii universally grow with $\left\langle d N_{c h} / d \eta\right\rangle^{1 / 3}$ at both energies and all $k_{\mathrm{T}}$. Only for $R_{\text {out }}$ at high $k_{\mathrm{T}}$ a less trivial behavior is observed, where the radii do not change within systematic error.

The most complicated behavior is observed as a function of $k_{\mathrm{T}}$. The $R_{\text {out }}$ at low multiplicity is flat with $k_{\mathrm{T}}$, but develops a very significant slope at the highest multiplicity - the radius changes by a factor of 2 between lowest and highest $k_{\mathrm{T}}$. For $R_{\text {side }}$ the situation is similar. At lowest multiplicity the dependence is flat and develops a slope at high $k_{\mathrm{T}}$. This slope is however much smaller than for $R_{\text {out }}$. As a result a particularly interesting behavior is observed for the $R_{\text {out }} / R_{\text {side }}$ ratio, which was the most sensitive probe of the freeze-out pattern in heavy-ion collisions. At lowest multiplicity it is large and flat with $k_{\mathrm{T}}$, for Gaussian radii it is consistent with 1.0. These however are collisions that are most different than heavy-ion ones. If one considers larger multiplicity, which in principle should make the collision more "heavy-ion like", the $R_{\text {out }} / R_{\text {side }}$ ratio decreases significantly and reaches values of 0.5 , much lower than for heavy-ion collisions. So at a first glance the behaviour of transverse radii exhibits some features qualitatively similar to heavy-ions, however detailed analysis, in three dimensions and as a function of $\left\langle d N_{c h} / d \eta\right\rangle^{1 / 3}$ shows striking differences between elementary and large systems. It is also clear that no "scaling" between $p p$ and $\mathrm{Pb}-\mathrm{Pb}$ data, similar to that reported by STAR, can be claimed here. The $R_{\text {long }}$ radius decreases with $k_{\mathrm{T}}$ at all multiplicities in a way similar to that observed in heavier systems. It shows that longitudinal dynamics may be similar in all systems.

The dependence of radii on $\left\langle d N_{c h} / d \eta\right\rangle^{1 / 3}$ can also be studied. It reveals that all three radii scale linearly with $\left\langle d N_{c h} / d \eta\right\rangle^{1 / 3}$, in all $k_{\mathrm{T}}$ ranges. This scaling is preserved across collision energies different by an order of magnitude. When compared to heavy-ion data however, the slope of such dependence is not the same. Therefore a trivial scaling of femtoscopic radii with $\left\langle d N_{c h} / d \eta\right\rangle^{1 / 3}$ is not valid for systems with different initial conditions.

In summary detailed analysis of differential pion femtoscopy radii in $p p$ collisions, as a function of $\vec{q}$ components in LCMS, pair $k_{\mathrm{T}}$, event multiplicity $\left\langle d N_{c h} / d \eta\right\rangle^{1 / 3}$ and collision energy shows interesting and non-trivial features that are not fully understood theoretically. In particular it seems that a hydrodynamic framework, which is successful in describing heavy-ion results is not applicable for the $p p$ system with similar assumptions. Nevertheless intriguing possibility for collectivity in $p p$ collisions [20] is still under debate and could be clarified with intense theoretical effort.

\section{Conclusions}

The pion femtoscopic measurements at the LHC have been presented. Results have been discussed for central $\mathrm{Pb}-\mathrm{Pb}$ collisions at $\sqrt{s_{N N}}=2.76 \mathrm{ATeV}$ as well as for $p p$ collisions at all multiplities and two collision energies: $\sqrt{s}=0.9$ and $7 \mathrm{TeV}$. Data for heavy ions are interpreted in the frame of hydrodynamic model. The model predicts both qualitatively as well as quantitatively the behavior of the radii. In particular it shows strong influence of radial flow and a change of the freeze-out pattern, when compared to central collisions at $\sqrt{s_{N N}}=200 \mathrm{AGeV}$ at RHIC. In $p p$ collisions a differential measurement was done versus $\left\langle d N_{c h} / d \eta\right\rangle^{1 / 3}$ and $k_{\mathrm{T}}$. Linear scaling with the former variable was observed, however with different scaling parameters than those observed in heavy-ion collisions. Significant dependence of radii on $k_{\mathrm{T}}$ was also observed, but the dependence of the slope on multiplicity is not trivial and qualitatively different than in heavy-ion collisions. In summary the two systems present different behavior and the physics interpretation of the radii in $p p$ collisions is still an open issue. 
EPJ Web of Conferences

\section{Acknowledgements}

This work has been financed by the Polish National Science Centre under decisions no. DEC2011/01/B/ST2/03483, DEC-2012/05/N/ST2/02757.

\section{References}

[1] V. Khachatryan et al. (CMS Collaboration), JHEP 1105, 029 (2011)

[2] K. Aamodt et al. (ALICE), JINST 3, S08002 (2008)

[3] K. Aamodt et al. (ALICE), Phys. Rev. D82, 052001 (2010), 1007.0516

[4] K. Aamodt et al. (ALICE Collaboration), Phys.Rev. D84, 112004 (2011), 1101.3665

[5] K. Aamodt et al. (ALICE Collaboration), Phys.Lett. B696, 328 (2011), 1012.4035

[6] B. Abelev et al. (ALICE Collaboration), Phys.Lett. B717, 151 (2012)

[7] W. Kittel, Acta Phys. Polon. B32, 3927 (2001), hep-ph/0110088

[8] G. Alexander, Rept. Prog. Phys. 66, 481 (2003), hep-ph/0302130

[9] M. Lisa, S. Pratt, R. Soltz, U. Wiedemann, Ann. Rev. Nucl. Part. Sci. 55, 311 (2005), nucl-ex/0505014

[10] M. Aggarwal et al. (STAR Collaboration), Phys.Rev. C83, 064905 (2011), 1004.0925

[11] B.I. Abelev et al. (STAR), Phys. Rev. C80, 024905 (2009), 0903. 1296

[12] K. Adcox et al. (PHENIX), Phys. Rev. Lett. 88, 192302 (2002), nucl-ex/0201008

[13] B.B. Back et al. (PHOBOS), Phys. Rev. C73, 031901 (2006), nucl-ex/0409001

[14] A. Kisiel, W. Broniowski, M. Chojnacki, W. Florkowski, Phys.Rev. C79, 014902 (2009), 0808.3363

[15] A.N. Makhlin, Y.M. Sinyukov, Z. Phys. C39, 69 (1988)

[16] S.V. Akkelin, Y.M. Sinyukov, Phys. Lett. B356, 525 (1995)

[17] S. Pratt, Nucl. Phys. A830, 51c (2009), 0907. 1094

[18] Z. Chajecki (STAR), AIP Conf. Proc. 828, 566 (2006)

[19] P. Bozek, Phys.Rev. C83, 044910 (2011), 1012 . 5927

[20] A. Kisiel, Phys.Rev. C84, 044913 (2011), 1012.1517 